\title{
CONFORMAL NETS ARE FACTORIZATION ALGEBRAS
}

\author{
ANDRÉ HENRIQUES
}

\begin{abstract}
We prove that conformal nets of finite index are an instance of the notion of a factorization algebra. This result is an ingredient in our proof that, for $G=S U(n)$, the Drinfel'd center of the category of positive energy representations of the based loop group is equivalent to the category of positive energy representations of the free loop group.
\end{abstract}

\section{INTRODUCTION}

In this note, we prove that conformal nets of finite index (Definitions 1.1 and 3.1 in [2]) form an instance of the notion of a factorization algebra. Our main result, Theorem 2] is a key ingredient in the proof, announced in [8, that the category of solitons of a finite index conformal net is a bicommutant category.

Our main theorem is an analog, within the coordinate-free setup of [2, of the additivity property of conformal nets. Let $\mathcal{A}$ be a conformal net on $S^{1}$, let $I \subset S^{1}$ be a closed interval, and let $\left\{I_{i} \subset I\right\}$ be collection of closed intervals whose interiors cover that of $I$. Additivity is the statement that the von Neumann algebras $\mathcal{A}\left(I_{i}\right)$ then generate a dense subalgebra in $\mathcal{A}(I)$ :

$$
\bigcup \stackrel{\circ}{I}_{i}=\stackrel{\circ}{I} \quad \Rightarrow \quad \bigvee \mathcal{A}\left(I_{i}\right)=\mathcal{A}(I) \quad \text { (additivity) }
$$

The additivity property of chiral conformal nets was proven in [5]. If one takes finitely many intervals $I_{i}$ whose union is $I$, then the corresponding property is called strong additivity:

$$
\bigcup I_{i}=I \quad \Rightarrow \quad \bigvee \mathcal{A}\left(I_{i}\right)=\mathcal{A}(I) . \quad \text { (strong additivity) }
$$

It is a result of Longo-Xu that chiral conformal nets of finite index satisfy strong additivity [12, $\S 5]$.

Let now $I$ be an abstract interval, and $\left\{I_{i} \subset I\right\}$ a finite collection of multiintervals (a multi-interval is a finite disjoint union of intervals) satisfying $\bigcup I_{i} \times I_{i}=$ $I \times I$. Equivalently, this is the requirement that for every pair of points $p, q \in I$ there exists an element of the cover that contains both $p$ and $q$. In Theorem 2, we prove that for every coordinate free conformal net $\mathcal{A}$ of finite index, not necessarily chira! 1 , we have:

$$
\bigcup I_{i} \times I_{i}=I \times I \quad \Rightarrow \quad \operatorname{colim} \mathcal{A}\left(I_{i}\right)=\mathcal{A}(I) . \quad \text { (factorization algebra) }
$$

The colimit which appears in the right hand side, informally denoted colim $\mathcal{A}\left(I_{i}\right)$, is that of a diagram involving the algebras $\mathcal{A}\left(I_{i}\right)$ and $\mathcal{A}\left(I_{i} \cap I_{j}\right)$. (The colimit is defined by a universal property in the category of von Neumann algebras and normal $*$-homomorphisms.) That diagram is written out in the left hand side of

\footnotetext{
${ }^{1} \mathrm{~A}$ coordinate-free conformal net is called chiral if the action of the rotation group on its vacuum sector has positive energy.
} 
equation (11), below. Here, the equation "colim $\mathcal{A}\left(I_{i}\right)=\mathcal{A}(I)$ " is the statement that the natural inclusions $\mathcal{A}\left(I_{i}\right) \rightarrow \mathcal{A}(I)$ extend to an isomorphism colim $\mathcal{A}\left(I_{i}\right) \rightarrow \mathcal{A}(I)$.

Remark. The category of von Neumann algebras and normal $*$-homomorphisms is cocomplete [11, Prop. 5.7] (see also [7, §7]).

\section{FACTORIZATION ALGEBRAS}

Let $\mathrm{Man}^{n}$ be the category whose objects are $n$-dimensional manifolds and whose morphisms are embeddings. We equip it with the symmetric monoidal structure given by disjoint union. A collection of open subsets $\left\{U_{i} \subset M\right\}$ of a manifold $M$ is a Weiss cover if for every finite subset $S \subset M$, there exists an index $i$ such that $S \subset U_{i}$ [4 , Chapt. 6]. Equivalently, being a Weiss cover means that for every $n \in \mathbb{N}$, the condition $\bigcup U_{i}^{n}=M^{n}$ is satisfied. Let $\mathrm{C}$ be a symmetric monoidal category.

Definition (4, Chapt.6]). An n-dimensional C-valued factorization algebra is a symmetric monoidal functor $\mathcal{A}: \mathrm{Man}^{n} \rightarrow \mathrm{C}$ which is a co-sheaf with respect to Weiss covers.

Here, being a co-sheaf with respect to Weiss covers means that, for every Weiss cover $\left\{U_{i} \subset M\right\}$, the natural map

$$
\operatorname{colim}\left(\begin{array}{c}
\mathcal{A}\left(U_{1} \cap U_{2}\right) \rightleftharpoons \\
\mathcal{A}\left(U_{1} \cap U_{3}\right) \rightleftharpoons \mathcal{A}\left(U_{1}\right) \\
\mathcal{A}\left(U_{2} \cap U_{3}\right) \rightleftharpoons \mathcal{P}\left(U_{2}\right) \\
\mathcal{A}\left(U_{1} \cap U_{4}\right) \rightleftharpoons \mathcal{A}\left(U_{3}\right) \\
\mathcal{A}\left(U_{2} \cap U_{4}\right) \\
\vdots
\end{array}\right.
$$

is an isomorphism. For later notational convenience, we abbreviate the left hand side of (11) as $\operatorname{colim}\left(\left\{\mathcal{A}\left(U_{i} \cap U_{j}\right)\right\} \rightrightarrows\left\{\mathcal{A}\left(U_{i}\right)\right\}\right)$.

In this paper, we are interested in 1-dimensional factorization algebras (or rather, a small variant of the notion of 1-dimensional factorization algebra) with values in the category of von Neumann algebras and normal *-homomorphisms.

An interval is an oriented 1-manifold diffeomorphic to [0,1]. A multi-interval is a finite disjoint union of intervals. Let INT* be the category whose objects are multi-intervals and whose morphisms are orientation preserving embeddings, and let INT $\subset$ INT $^{*}$ be its full subcategory of intervals. Let VN denote the category of von Neumann algebras and normal $*$-homomorphisms, equipped with the symmetric monoidal structure given by spatial tensor product. By the split property [2, Def. 1.1], a conformal net can be viewed as a symmetric monoidal functor $\mathcal{A}: \mathrm{INT}^{*} \rightarrow \mathrm{VN}$.

We introduce a variant of the notion of Weiss cover that accounts for the fact that morphisms in INT* are not open but rather closed inclusions. Given a topological space $X$, a Weiss c-cover is a family of closed subsets $\left\{V_{i} \subset X\right\}$ that satisfies $\cup \stackrel{\circ}{V}_{i}^{n}=X^{n}$ for every $n \in \mathbb{N}$. Here, $\stackrel{\circ}{V}_{i}$ denotes the relative interior of $V_{i}$ inside $X$, i.e., the largest subset of $V_{i}$ which is open in $X$. (For example, the relative interior of $[0,1]$ inside $[0,2]$ is the half-open interval $[0,1[)$.

Throughout this paper, all conformal nets are assumed irreducible, i.e., all the algebras $\mathcal{A}(I)$ are assumed to be factors (we work with conformal nets in the sense 
of [2, Def. 1.1]). The following statement expresses the idea that conformal nets are factorization algebras:

Theorem 1. Let $\mathcal{A}:$ INT $^{*} \rightarrow \mathrm{VN}$ be a conformal net of finite index. Then $\mathcal{A}$ is a co-sheaf with respect to Weiss c-covers. Namely, for every multi-interval I, and every Weiss c-cover of $I$ by multi-intervals $I_{i} \subset I$, the natural map

$$
q: \operatorname{colim}\left(\left\{\mathcal{A}\left(I_{i} \cap I_{j}\right)\right\} \rightrightarrows\left\{\mathcal{A}\left(I_{i}\right)\right\}\right) \rightarrow \mathcal{A}(I)
$$

is an isomorphism.

Remark. Here, it is crucial to use covers by closed multi-intervals. For a chiral conformal net $\mathcal{A}$ on $S^{1}$, the functor that sends an open multi-interval $U$ to the algebra $\mathcal{A}(\bar{U})$ is never a factorisation algebra, unless $\mathcal{A}$ is trivial ( $\bar{U}$ denotes the closure of $U$ in $S^{1}$ ), because the map

$$
A:=\operatorname{colim}_{\varepsilon \rightarrow 0} \mathcal{A}([\varepsilon, 1-\varepsilon]) \rightarrow \mathcal{A}([0,1])
$$

is not an isomorphism. Let $I_{\varepsilon} \subset S^{1}$ be the image of $[\varepsilon, 1-\varepsilon]$ under the exponential map $t \mapsto e^{2 \pi i t}: \mathbb{R} \rightarrow S^{1}$. The obvious isomorphisms $\mathcal{A}([\varepsilon, 1-\varepsilon]) \rightarrow \mathcal{A}\left(I_{\varepsilon}\right)$ followed by the standard actions of $\mathcal{A}\left(I_{\varepsilon}\right)$ on the vacuum Hilbert space $H_{0}$ yield an irreducible representation of $A$ on $H_{0}$. However, $\mathcal{A}([0,1])$ is a $I I I_{1}$ factor, which admits no irreducible representations [6. Thm. 2.13].

We can sharpen the above result a little bit. Given a compact topological space $X$, a collection $\left\{V_{i} \subset X\right\}_{i \in \mathcal{I}}$ of closed subsets is called a 2-cover if there exists a finite subset $\mathcal{I}^{\prime} \subset \mathcal{I}$ such that $\bigcup_{i \in \mathcal{I}^{\prime}} V_{i}^{2}=X^{2}$. Any Weiss $c$-cover is a 2-cover, and Theorem 1 is a formal consequence of the following stronger result:

Theorem 1'. Let $\mathcal{A}$ be a conformal net of finite index, let I be a multi-interval, and let $\left\{I_{i} \subset I\right\}$ be a 2-cover by multi-intervals. Assume furthermore that there exists an element of the 2 -cover that contains $\partial I$. Then the natural map

$$
q: \operatorname{colim}\left(\left\{\mathcal{A}\left(I_{i} \cap I_{j}\right)\right\} \rightrightarrows\left\{\mathcal{A}\left(I_{i}\right)\right\}\right) \rightarrow \mathcal{A}(I)
$$

is an isomorphism.

When $I$ is connected, the statement of Theorem 1 simplifies:

Theorem 2. Let $\mathcal{A}$ be a conformal net of finite index, let $I$ be an interval, and let $\left\{I_{i} \subset I\right\}$ be a 2-cover by multi-intervals. Then the natural map

$$
q: \operatorname{colim}\left(\left\{\mathcal{A}\left(I_{i} \cap I_{j}\right)\right\} \rightrightarrows\left\{\mathcal{A}\left(I_{i}\right)\right\}\right) \rightarrow \mathcal{A}(I)
$$

is an isomorphism.

\section{Proofs}

In this section, we present the proofs of the above theorems. We first prove Theorem 2 . We then prove Theorem 10 by a slight variation of the argument. Theorem 1 is then a formal consequence of Theorem 11. We begin with some lemmas. We first note that, when working with multi-intervals, a 2-cover induces a cover in the usual sense:

Lemma 3. Let $I$ be a multi-interval and let $\left\{I_{i} \subset I\right\}_{i \in \mathcal{I}}$ be a 2-cover by multiintervals. Then $\bigcup \stackrel{\circ}{I}_{i}=\stackrel{\circ}{I}$. 
Proof. By definition, $\bigcup_{i \in \mathcal{I}^{\prime}} I_{i}^{2}=I^{2}$ for some finite subset $\mathcal{I}^{\prime} \subset \mathcal{I}$. Given a point $p \in \stackrel{I}{I}$, pick sequences $\left(x_{n}\right)$ and $\left(y_{n}\right)$ in $I$ converging to $p$ and satisfying $x_{n}<p<y_{n}$. For every $n$, there exists an index $i \in \mathcal{I}^{\prime}$ such that $x_{n}$ and $y_{n}$ are both in $I_{i}$. The set $\mathcal{I}^{\prime}$ being finite, there exists an $I_{i}$ that contains infinitely many $x_{n}$ 's and $y_{n}$ 's. Since $I_{i}$ is a multi-interval, it contains $p$ in its interior.

The next lemma is technical in nature. It is a generalisation of [2, Lem. 1.9]. Let $\mathcal{A}$ be a conformal net (not necessarily of finite index) and let $I$ be a multi-interval:

Lemma 4. Let $\mathcal{I}=\left\{I_{i} \subset I\right\}$ be a collection of multi-intervals satisfying $\bigcup \stackrel{\circ}{I}_{i}=\stackrel{\circ}{\text {. }}$. Let $\varphi \in \operatorname{Diff}(I)$ be a diffeomorphism in the connected component of the identity, and let $\hat{I}:=\varphi\left(I_{0}\right)$ for some $I_{0} \in \mathcal{I}$. Let $H$ be a Hilbert space equipped with actions $\rho_{i}: \mathcal{A}\left(I_{i}\right) \rightarrow B(H)$ satisfying

(1) $\left.\rho_{i}\right|_{\mathcal{A}\left(I_{i} \cap I_{j}\right)}=\left.\rho_{j}\right|_{\mathcal{A}\left(I_{i} \cap I_{j}\right)}: \mathcal{A}\left(I_{i} \cap I_{j}\right) \rightarrow B(H)$.

(2) For every $I_{j}, I_{k} \in \mathcal{I}$ and every intervals $J \subset I_{j}, K \subset I_{k}$ with disjoint interiors, the algebras $\rho_{j}(\mathcal{A}(J))$ and $\rho_{k}(\mathcal{A}(K))$ commute.

Then the actions $\left.\rho_{i}\right|_{\mathcal{A}\left(\hat{I} \cap I_{i}\right)}$ of $\mathcal{A}\left(\hat{I} \cap I_{i}\right)$ on $H$ extend (uniquely) to an action $\hat{\rho}: \mathcal{A}(\hat{I}) \rightarrow B(H)$.

Proof. We write $\rho_{0}$ for the action of $\mathcal{A}\left(I_{0}\right)$ on $H$. We may assume without loss of generality that $\varphi$ fixes a neighbourhood of $\partial I$. Provided that is the case, we can write $\varphi$ as a product of diffeomorphisms $\varphi=\varphi_{1} \circ \ldots \circ \varphi_{n}$ with $\operatorname{supp}\left(\varphi_{s}\right) \subset \check{I}_{i_{s}}$ for some $I_{i_{s}} \in \mathcal{I}$. Let $u_{s} \in \mathcal{A}\left(I_{i_{s}}\right)$ be unitaries s.t. $\operatorname{Ad}\left(u_{s}\right)=\mathcal{A}\left(\varphi_{s}\right)$ [2, Def. 1.1 (iv)]. Identifying the elements $u_{s}$ with their images in $B(H)$, we set

$$
\hat{\rho}(a):=u_{1} \ldots u_{n} \rho_{0}\left(\mathcal{A}\left(\varphi^{-1}\right)(a)\right) u_{n}^{*} \ldots u_{1}^{*} .
$$

For every $I_{\ell} \in \mathcal{I}$ and every sufficiently small interval $K \subset \hat{I} \cap I_{\ell}$, we will show that

$$
\left.\hat{\rho}\right|_{\mathcal{A}(K)}=\left.\rho_{\ell}\right|_{\mathcal{A}(K)} \text {. }
$$

Here, 'sufficiently small' means that the intervals $K_{s}:=\varphi_{s}^{-1}\left(\ldots\left(\varphi_{1}^{-1}(K)\right)\right)$ are contained in $I_{k_{s}}$ for some $I_{k_{s}} \in \mathcal{I}$, and that for every $s^{\prime} \leq n$ either $K_{s} \subset I_{i_{s^{\prime}}}$ or $K_{s} \cap \operatorname{supp}\left(\varphi_{s^{\prime}}\right)=\emptyset$.

For every $s \leq n$, we claim that

$$
u_{1} \ldots u_{s} \rho_{k_{s}}\left(\mathcal{A}\left(\varphi_{s}^{-1} \circ \ldots \circ \varphi_{1}^{-1}\right)(a)\right) u_{s}^{*} \ldots u_{1}^{*}=\rho_{\ell}(a) \quad \forall a \in \mathcal{A}(K) .
$$

Equation (2) is the special case $s=n$. We prove (3) by induction on $s$. The base case $\left(s=0, k_{0}=\ell\right)$ is trivial. The induction step reduces to the equation

$$
\rho_{k_{s}}\left(\mathcal{A}\left(\varphi_{s}^{-1}\right)(b)\right)=u_{s}^{*} \rho_{k_{s-1}}(b) u_{s},
$$

with $b=\mathcal{A}\left(\varphi_{s-1}^{-1} \circ \ldots \circ \varphi_{1}^{-1}\right)(a)$. Recall that $b \in \mathcal{A}\left(K_{s-1}\right), u_{s} \in \mathcal{A}\left(I_{i_{s}}\right)$ and that, by assumption, either $K_{s-1} \subset I_{i_{s}}$ or $K_{s-1} \cap \operatorname{supp}\left(\varphi_{s}\right)=\emptyset$. In the first case, we have

$$
u_{s}^{*} \rho_{k_{s-1}}(b) u_{s}=u_{s}^{*} \rho_{i_{s}}(b) u_{s}=\rho_{i_{s}}\left(u_{s}^{*} b u_{s}\right)=\rho_{i_{s}}\left(\mathcal{A}\left(\varphi_{s}^{-1}\right)(b)\right)=\rho_{k_{s}}\left(\mathcal{A}\left(\varphi_{s}^{-1}\right)(b)\right) .
$$

In the second case, the elements $\rho_{k_{s-1}}(b)$ and $u_{s}$ commute:

$$
u_{s}^{*} \rho_{k_{s-1}}(b) u_{s}=\rho_{k_{s-1}}(b)=\rho_{k_{s}}(b)=\rho_{k_{s}}\left(\mathcal{A}\left(\varphi_{s}^{-1}\right)(b)\right),
$$

where the last equality holds since $b \in A\left(K_{s-1}\right)$ and $\varphi_{s}$ acts trivially on $K_{s-1}$. This finishes the proof of (3) and hence of (2). Finally, by strong additivity (which is one of the axioms in [2, Def. 1.1]), it follows from (2) that $\hat{\rho}(a)=\rho_{\ell}(a)$ for every $a \in \mathcal{A}\left(\hat{I} \cap I_{\ell}\right)$. 
We now establish some assumptions under which the hypotheses of Lemma 4 are satisfied:

Lemma 5. Let $I$ be a multi-interval, and let $\mathcal{I}=\left\{I_{i} \subset I\right\}$ be a 2-cover. Let $\rho_{i}: \mathcal{A}\left(I_{i}\right) \rightarrow B(H)$ be actions satisfying $\left.\rho_{i}\right|_{\mathcal{A}\left(I_{i} \cap I_{j}\right)}=\left.\rho_{j}\right|_{\mathcal{A}\left(I_{i} \cap I_{j}\right)}$. Then, for every $I_{j}, I_{k} \in \mathcal{I}$ and every intervals $J \subset I_{j}, K \subset I_{k}$ with disjoint interiors, we have

$$
\left[\rho_{j}(\mathcal{A}(J)), \rho_{k}(\mathcal{A}(K))\right]=0 .
$$

Proof. We assume without loss of generality that the 2 -cover is finite. The finite set $S:=\bigcup_{i \in \mathcal{I}} \partial I_{i}$ decomposes $J$ and $K$ into a finitely many intervals: $J=J_{1} \cup \ldots \cup J_{n}$ and $K=K_{1} \cup \ldots \cup K_{m}$. For each pair $J_{r}, K_{s}$ of above intervals, we will argue that

$$
\left[\rho_{j}\left(\mathcal{A}\left(J_{r}\right)\right), \rho_{k}\left(\mathcal{A}\left(K_{s}\right)\right)\right]=0 .
$$

Pick interior points $x \in \stackrel{\circ}{J}_{r}$ and $y \in \stackrel{\circ}{K}_{s}$. Since $\mathcal{I}$ is a 2-cover, there exists an $i \in \mathcal{I}$ such that $\{x, y\} \subset I_{i}$. It follows that $J_{r} \cup K_{s} \subset I_{i}$. The actions of $\mathcal{A}\left(J_{r}\right)$ and $\mathcal{A}\left(K_{s}\right)$ on $H$ factor through that of $\mathcal{A}\left(I_{i}\right)$, so equation (4) follows.

Equation (4) being true for every pair $J_{r}, K_{s}$ as above, by strong additivity, it follows that the algebras $\rho_{j}(\mathcal{A}(J))=\bigvee_{r} \rho_{j}\left(\mathcal{A}\left(J_{r}\right)\right)$ and $\rho_{k}(\mathcal{A}(K))=\bigvee_{s} \rho_{k}\left(\mathcal{A}\left(K_{s}\right)\right)$ commute with each other.

The following lemma contains the main argument of the proof of Theorem 2 Let $\mathcal{A}$ be a conformal net of finite index:

Lemma 6. Let $I$ be an interval and let $\mathcal{I}=\left\{I_{i} \subset I\right\}$ be a 2 -cover. Let $H$ be a Hilbert space equipped with actions $\rho_{i}: \mathcal{A}\left(I_{i}\right) \rightarrow B(H)$ satisfying $\left.\rho_{i}\right|_{\mathcal{A}\left(I_{i} \cap I_{j}\right)}=\left.\rho_{j}\right|_{\mathcal{A}\left(I_{i} \cap I_{j}\right)}$. Then those maps extend to an action of $\mathcal{A}(I)$.

Proof. We may assume, without loss of generality, that the 2-cover is closed under taking subsets: $\left(I_{i} \in \mathcal{I}\right.$ and $J \subset I_{i}, J$ a multi-interval $) \Rightarrow(J \in \mathcal{I})$.

By Lemmas 3 and 5 , we are in a situation to apply Lemma 4 The latter implies that for every interval $J \varsubsetneqq I$, the actions of $\mathcal{A}\left(I_{i} \cap J\right)$ extend (uniquely) to an action of $\mathcal{A}(J)$. We may therefore assume without loss of generality that $I=[0,5]$, and that the 2-cover contains the multi-intervals $[0,2] \cup[3,5]$ and $[1,4]$ as elements.

Recall that $L^{2}(-)$ is the unit for the operation $\otimes$ of Connes fusion. We have $H \cong L^{2} \mathcal{A}([1,4]) \bigotimes_{\mathcal{A}([1,4])} H$, both as $\mathcal{A}([1,4])$-modules and as $\mathcal{A}([0,1] \cup[4,5])$ modules. By [3, Cor.2.9], the vacuum sector $L^{2} \mathcal{A}([1,4])$ is isomorphic to

$$
L^{2} \mathcal{A}([2,3]) \bigotimes_{\mathcal{A}\left([2,3] \cup_{\{2,3\}}[\overline{2,3]})\right.}\left(L^{2} \mathcal{A}([1,4]) \bigotimes_{\mathcal{A}([1,2] \cup[3,4])} L^{2} \mathcal{A}([1,4])\right)
$$

as an $\mathcal{A}([1,4])-\mathcal{A}([1,4])$-bimodule (this is where we use the assumption that $\mathcal{A}$ has finite index). Here, $[\overline{2,3}$ denotes the interval $[2,3]$ equipped with the opposite orientation, and the algebra $\mathcal{A}\left([2,3] \cup_{\{2,3\}}[2,3]\right)$ associated to the circle $[2,3] \cup_{\{2,3\}}[\overline{2,3}]$ is described in [3, Prop. 1.25]. The action of $\mathcal{A}\left([2,3] \cup_{\{2,3\}}[\overline{2,3}]\right)$ on $L^{2} \mathcal{A}([1,4]) \bigotimes_{\mathcal{A}([1,2] \cup[3,4])} L^{2} \mathcal{A}([1,4])$ comes from the left action of $\mathcal{A}([2,3]) \subset \mathcal{A}([1,4])$ on the second copy of $L^{2} \mathcal{A}([1,4])$ and the right action of $\mathcal{A}([2,3]) \subset \mathcal{A}([1,4])$ on the first copy of $L^{2} \mathcal{A}([1,4])$.

Let us abbreviate $\mathcal{A}([a, b])$ by $\mathcal{A}_{a b}, \mathcal{A}([a, b] \cup[c, d])$ by $\mathcal{A}_{a b \cup c d}$, and $\mathcal{A}\left([a, b] \cup_{\{a, b\}}\right.$ $[\overline{a, b}])$ by $\mathcal{A}_{\widehat{a b}}$. We have:

$$
H \cong L^{2} \mathcal{A}_{14} \otimes_{\mathcal{A}_{14}} H \quad \text { and } \quad L^{2} \mathcal{A}_{14} \cong L^{2} \mathcal{A}_{23} \otimes_{\mathcal{A}_{\widehat{23}}}\left(L^{2} \mathcal{A}_{14} \otimes_{\mathcal{A}_{12 \cup 34}} L^{2} \mathcal{A}_{14}\right) .
$$


Combining those two facts, one gets

$$
\begin{aligned}
& H \cong L^{2} \mathcal{A}_{14} \otimes_{\mathcal{A}_{14}} H \\
& \cong\left(L^{2} \mathcal{A}_{23} \nabla_{\mathcal{A}_{23}}\left(L^{2} \mathcal{A}_{14} \bigotimes_{\mathcal{A}_{12 \cup 34}} L^{2} \mathcal{A}_{14}\right)\right) \bigotimes_{\mathcal{A}_{14}} H \\
& \cong L^{2} \mathcal{A}_{23} \otimes_{\mathcal{A}_{23}}\left(L^{2} \mathcal{A}_{14} \otimes_{\mathcal{A}_{12 \cup 34}} L^{2} \mathcal{A}_{14} \otimes_{\mathcal{A}_{14}} H\right) \\
& \cong L^{2} \mathcal{A}_{23} \nabla_{\mathcal{A}_{23}}\left(L^{2} \mathcal{A}_{14} \otimes_{\mathcal{A}_{12 \cup 34}} H\right) \text {. }
\end{aligned}
$$

Using that $H \cong L^{2} \mathcal{A}_{02 \cup 35} \otimes_{\mathcal{A}_{02 \cup 35}} H$ and the existence of a (non-canonical) isomorphism

$$
L^{2} \mathcal{A}_{14} \otimes_{\mathcal{A}_{12 \cup 34}} L^{2} \mathcal{A}_{02 \cup 35} \cong L^{2} \mathcal{A}_{02} \otimes_{\mathcal{A}_{12}^{o p}} L^{2} \mathcal{A}_{14} \otimes_{\mathcal{A}_{34}} L^{2} \mathcal{A}_{35} \cong L^{2} \mathcal{A}_{05}
$$

which is compatible with the left actions of $\mathcal{A}_{14}$ and $\mathcal{A}_{01 \cup 45}$ and the right actions of $\mathcal{A}_{02} \cup 35$ and $\mathcal{A}_{23}$ ([2, Cor. 1.33] and [3, Lem. A.4]), we get the following sequence of isomorphisms of $\mathcal{A}_{14^{-}}$and $\mathcal{A}_{01 \cup 45^{-}}$-modules:

$$
\begin{aligned}
H & \cong L^{2} \mathcal{A}_{23} \otimes_{\mathcal{A}_{\widehat{23}}}\left(L^{2} \mathcal{A}_{14} \otimes_{\mathcal{A}_{12 \cup 34}} H\right) \\
& \cong L^{2} \mathcal{A}_{23} \otimes_{\mathcal{A}_{\widehat{23}}}\left(L^{2} \mathcal{A}_{14} \otimes_{\mathcal{A}_{12 \cup 34}} L^{2} \mathcal{A}_{02 \cup 35} \otimes_{\mathcal{A}_{02 \cup 35}} H\right) \\
& \cong L^{2} \mathcal{A}_{23} \otimes_{\mathcal{A}_{\widehat{23}}}\left(L^{2} \mathcal{A}_{05} \otimes_{\mathcal{A}_{02 \cup 35}} H\right) .
\end{aligned}
$$

The actions of $\mathcal{A}_{14}$ and of $\mathcal{A}_{01 \cup 45}$ on

$$
L^{2} \mathcal{A}_{23} \otimes_{\mathcal{A}_{\overparen{23}}}\left(L^{2} \mathcal{A}_{05} \otimes_{\mathcal{A}_{02 \cup 35}} H\right)
$$

extend to an action of $\mathcal{A}_{05}$ because they both act on $L^{2} \mathcal{A}_{05}$. The actions of $\mathcal{A}_{14}$ and of $\mathcal{A}_{01 \cup 45}$ on $H$ therefore also extend to an action of $\mathcal{A}_{05}$.

To help the reader digest the argument in the above proof, we include a graphical rendering of the isomorphisms which appear in (5) and (6):

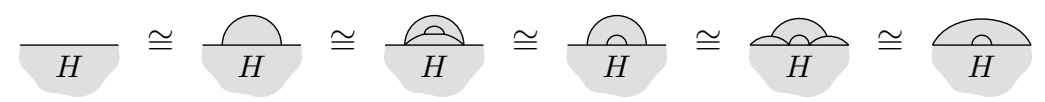

With Lemma 6 in place, the proof of Theorem 2 is now easy:

Proof of Theorem 2 . We first note that, by the strong additivity property of conformal nets [2, Def. 1.1], the map $q$ has dense image. It is therefore surjective, as any morphism of von Neumann algebras whose image is dense is automatically surjective [13, Chapt. III, Prop. 3.12].

To show that $q$ is injective, pick a faithful representation

$$
\pi: \operatorname{colim}\left(\left\{\mathcal{A}\left(I_{i} \cap I_{j}\right)\right\} \rightrightarrows\left\{\mathcal{A}\left(I_{i}\right)\right\}\right) \rightarrow B(H)
$$

and let $\rho_{i}:=\left.\pi\right|_{\mathcal{A}\left(I_{i}\right)}$. By Lemma [6, this extends to an action $\rho: \mathcal{A}(I) \rightarrow B(H)$. As $\pi$ is injective and $\pi=\rho \circ q$, the map $q$ is also injective.

The proof of Theorem 1 follows closely that of Theorem 2 .

Proof of Theorem 11. Let $I$ be a multi-interval, and let $\mathcal{I}=\left\{I_{i} \subset I\right\}$ be a 2-cover one of whose elements contains $\partial I$. As in the proof of Theorem 2, it is enough to argue that if $H$ is a Hilbert space equipped with actions $\rho_{i}: \mathcal{A}\left(I_{i}\right) \rightarrow B(H)$ satisfying $\left.\rho_{i}\right|_{\mathcal{A}\left(I_{i} \cap I_{j}\right)}=\left.\rho_{j}\right|_{\mathcal{A}\left(I_{i} \cap I_{j}\right)}$, then those extend to an action of $\mathcal{A}(I)$.

Without loss of generality, we may assume that the 2-cover is closed under taking subsets: $\left(I_{i} \in \mathcal{I}\right.$ and $\left.J \subset I_{i}\right) \Rightarrow(J \in \mathcal{I})$. In particular, we may assume that the 
2-cover admits an element which doesn't intersect its boundary, and which has exactly one connected component in each connected component on $I$.

By Lemmas 3 and 5, we are in a situation to apply Lemma 4, We can therefore assume, without loss of generality, that $I=\bigsqcup_{k=1}^{n}[0,5]$, and that $\mathcal{I}$ contains the multi-intervals $\bigsqcup_{k=1}^{n}([0,2] \cup[3,5])$ and $\bigsqcup_{k=1}^{n}[1,4]$ as elements.

Following the structure of the proof of Lemma 6, we have isomorphisms:

$$
\begin{aligned}
& H \cong L^{2}\left(\mathcal{A}_{14}\right)^{\otimes n} \otimes_{\mathcal{A}_{14}^{\otimes n}} H \\
& \cong\left(L^{2}\left(\mathcal{A}_{23}\right)^{\otimes n} \bigotimes_{\mathcal{A}_{23}^{\otimes n}}\left(L^{2}\left(\mathcal{A}_{14}\right)^{\otimes n} \bigotimes_{\mathcal{A}_{12 \cup 34}^{\otimes n}} L^{2}\left(\mathcal{A}_{14}\right)^{\otimes n}\right)\right) \bigotimes_{\mathcal{A}_{14}^{\otimes n}} H \\
& \cong L^{2}\left(\mathcal{A}_{23}\right)^{\otimes n} \bigotimes_{\mathcal{A}_{23}^{\otimes n}}\left(L^{2}\left(\mathcal{A}_{14}\right)^{\otimes n} \bigotimes_{\mathcal{A}_{12 \cup 34}^{\otimes n}} L^{2}\left(\mathcal{A}_{14}\right)^{\otimes n} \bigotimes_{\mathcal{A}_{14}^{\otimes n}} H\right) \\
& \cong L^{2}\left(\mathcal{A}_{23}\right)^{\otimes n} \otimes_{\mathcal{A}_{23}^{\otimes n}}\left(L^{2}\left(\mathcal{A}_{14}\right)^{\otimes n} \otimes_{\mathcal{A}_{12 \cup 34}^{\otimes n}} H\right) \text {. } \\
& \cong L^{2}\left(\mathcal{A}_{23}\right)^{\otimes n} \nabla_{\mathcal{A}_{23}^{\otimes n}}\left(L^{2}\left(\mathcal{A}_{14}\right)^{\otimes n} \nabla_{\mathcal{A}_{12 \cup 34}^{\otimes n}} L^{2}\left(\mathcal{A}_{02 \cup 35}\right)^{\otimes n} \nabla_{\mathcal{A}_{02 \cup 35}^{\otimes n}} H\right) \\
& \cong L^{2}\left(\mathcal{A}_{23}\right)^{\otimes n} \otimes_{\mathcal{A}_{23}^{\otimes n}}\left(L^{2}\left(\mathcal{A}_{05}\right)^{\otimes n} \otimes_{\mathcal{A}_{02 \cup 35}^{\otimes n}} H\right)
\end{aligned}
$$

of $\mathcal{A}\left(\bigsqcup^{n}([0,2] \cup[3,5])\right)$ - and $\mathcal{A}\left(\bigsqcup^{n}[1,4]\right)$-modules.

The actions of $\mathcal{A}\left(\bigsqcup^{n}([0,2] \cup[3,5])\right)$ and of $\mathcal{A}\left(\bigsqcup^{n}[1,4]\right)$ on the Hilbert space $L^{2}\left(\bigotimes^{n} \mathcal{A}_{23}\right) \bigotimes_{\mathcal{A}_{23}^{\otimes n}}\left(L^{2}\left(\bigotimes^{n} \mathcal{A}_{05}\right) \bigotimes_{\bigotimes^{n} \mathcal{A}_{02 \cup 35}} H\right)$ visibly extend to an action of the von Neumann algebra $\mathcal{A}\left(\bigsqcup^{n}[0,5]\right)=\mathcal{A}(I)$. They therefore also extend to an action of $\mathcal{A}(I)$ on $H$.

\section{An Application}

In our recent preprint [8], we introduced higher categorical analogs of von Neumann algebras called bicommutant categories. A bicommutant category is a tensor category which is equivalent to its bicommutant inside $\operatorname{Bim}(R)$. (The latter is the category of all bimodules over a hyperfinite factor; it plays the role of the algebra of bounded operators on a Hilbert space.) A bicommutant category is also equipped with a higher categorical analog of a $*$-structure, called a bi-involutive structure 9 , Def. 2.3].

In $[8$, we made the following announcement: for $G$ the group $S U(n)$ and for $k$ a positive integer, the category of positive energy representations of the based loop group of $G$ at level $k$ is a bicommutant category. Moreover, its Drinfel'd center is the category of positive energy representations of the free loop group of $G$ :

$$
Z\left(\operatorname{Rep}_{\substack{\text { pos. } \\ \text { en. }}}^{k}(\Omega G)\right)=\operatorname{Rep}_{\substack{\text { pos. } \\ \text { en. }}}^{k}(L G)
$$

We then argued that this result provides good evidence for our claim that the tensor category of positive energy representation of the based loop group is the value of Chern-Simons theory on a point.

Remark 7. The tensor category of positive energy representations of $L G$, as defined using conformal nets (see, e.g., [6, 15]), has, to our knowledge, not been compared to the corresponding tensor category defined using affine Lie algebras (or vertex algebras, or quantum groups). The right hand side of (77) refers to the tensor category defined in 15 . 
Remark 8. We expect the relation (17) to hold true for every compact connected Lie group $G$ and every level $k \in H_{+}^{4}(B G, \mathbb{Z})$. It is conjectured by many people that all chiral WZW conformal nets have finite index (see [6] [14] 15] [2, §4.C] 10, §8] for the definition of these conformal nets in various degrees of generality). The finite index property is known when $G=S U(n)$ [16, 15], and in a few other cases. Our proof of (77) relies crucially on the fact that the chiral WZW conformal nets associated to $G$ have finite index. However, our dependence on this result is the only place where we use that $G$ is the group $S U(n)$.

We can generalize (7) to arbitrary conformal nets of finite index. The analog of the tensor category of positive energy representations of $\Omega G$ is the tensor category $\mathcal{T}_{\mathcal{A}}$ of solitons of the conformal net $\mathcal{A}[12, \S 3.0 .1]$. The claim is then that the Drinfel'd center of the tensor category of solitons of $\mathcal{A}$ is the braided tensor category of representations of $\mathcal{A}$.

By definition, a soliton is a Hilbert space equipped with compatible actions of the algebras $\mathcal{A}(I)$, where $I$ ranges over all subintervals of the standard circle whose interior does not contain the base point $1 \in S^{1}$. Equivalently, it ranges over all subintervals $I \subsetneq S_{\text {cut }}^{1}$, where $S_{\text {cut }}^{1}$ is the manifold obtained from the standard circle by removing its base point and replacing it by two points:

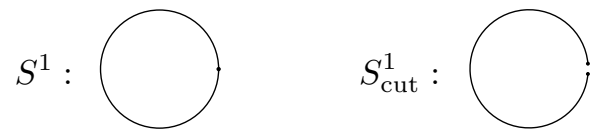

The monoidal structure on $\mathcal{T}_{\mathcal{A}}$ is defined as follows. Let $H$ and $K$ be two solitons. Let $I_{+}$be the upper half of $S^{1}$, and let $I_{-}$be its lower half. Precomposing the left action of $\mathcal{A}\left(I_{-}\right)$on $H$ by the map

$$
\mathcal{A}\left(z \mapsto \bar{z}: I_{+} \rightarrow I_{-}\right): \mathcal{A}\left(I_{+}\right)^{o p} \rightarrow \mathcal{A}\left(I_{-}\right)
$$

yields a right action of $\mathcal{A}\left(I_{+}\right)$on $H$. We let

$$
H \otimes K:=H \otimes_{\mathcal{A}\left(I_{+}\right)} K .
$$

Here, $\mathcal{A}\left(I_{+}\right)$acts on $K$ in the usual way, and acts on $H$ on the right via the action described above. The left actions of $\mathcal{A}\left(I_{+}\right)$on $H$ and of $\mathcal{A}\left(I_{-}\right)$on $K$ induce corresponding actions on $H \otimes K$. Given an interval $J \subset S^{1}, 1 \notin J,-1 \in \stackrel{\circ}{J}$, then, by the same argument as in [2, Def. 1.31], the actions of $\mathcal{A}\left(J \cap I_{+}\right)$and $\mathcal{A}\left(J \cap I_{-}\right)$on $H \otimes K$ extend to an action of $\mathcal{A}(J)$. By Lemma 4, it follows that for every interval $J \subsetneq S_{\text {cut }}^{1}$, the actions of $\mathcal{A}\left(J \cap I_{+}\right)$and $\mathcal{A}\left(J \cap I_{-}\right)$on $H \otimes K$ extend to an action

$$
\rho_{J}: \mathcal{A}(J) \rightarrow B(H \otimes K) .
$$

All together, these actions equip $H \otimes K$ with the structure of a soliton.

Given a soliton $H$, with actions $\rho_{I}: \mathcal{A}(I) \rightarrow B(H)$ for $I \subsetneq S_{\text {cut }}^{1}$, its conjugate $\bar{H}$ is the complex conjugate Hilbert space equipped with the actions

$$
\mathcal{A}(I) \stackrel{\mathcal{A}(z \mapsto \bar{z})}{\longrightarrow} \mathcal{A}(\bar{I})^{o p} \stackrel{*}{\longrightarrow} \overline{\mathcal{A}(\bar{I})} \stackrel{\overline{\rho_{\bar{I}}}}{\longrightarrow} \overline{B(H)}=B(\bar{H}) .
$$

Here, $\bar{I}$ denotes the image of $I \subset S^{1}$ under the complex conjugation map $S^{1} \rightarrow S^{1}$. The conjugation on $\mathcal{T}_{\mathcal{A}}$ squares to the identity and satisfies $\overline{H \otimes K} \cong \bar{K} \otimes \bar{H}$.

Definition ([9, Def. 5.3]). An object $\Omega$ of a tensor category $(\mathcal{T}, \otimes)$ is called absorbing if it is non-zero and satisfies

$$
(X \neq 0) \Rightarrow(X \otimes \Omega \cong \Omega \cong \Omega \otimes X) \quad \forall X \in \mathcal{T} .
$$


Remark. If $\mathcal{T}$ admits a conjugation, it is a little bit easier to check that an object is absorbing. $\Omega \in \mathcal{T}$ is absorbing if it is non-zero and if $X \otimes \Omega \cong \Omega$ for every $X \neq 0$; see the comments after [9, Def. 5.3] for a proof.

The next result, about the existence of absorbing objects, is a key ingredient in the proof, announced in [8, that the category of solitons of a conformal net with finite index is a bicommutant category, and that its Drinfel'd center is the category of representations of $\mathcal{A}$. The proof relies on Lemma 6 (which is essentially equivalent to Theorem 2).

Given a non-trivial conformal net $\mathcal{A}$, let

$$
\Omega_{\mathcal{A}}:=L^{2}\left(\mathcal{A}\left(S_{\text {cut }}^{1}\right)\right) \in \mathcal{T}_{\mathcal{A}},
$$

with actions of $\mathcal{A}(I)$ for $I \subsetneq S_{\text {cut }}^{1}$ provided by the obvious inclusion $\mathcal{A}(I) \rightarrow \mathcal{A}\left(S_{\text {cut }}^{1}\right)$ followed by the left action of $\mathcal{A}\left(S_{\text {cut }}^{1}\right)$ on its $L^{2}$-space.

Alternatively, the soliton $\Omega_{\mathcal{A}}$ can be described as follows. Let its underlying Hilbert space be the vacuum Hilbert space $H_{0}$ of the conformal net $\mathcal{A}$. Given an interval $I=\left[e^{i a}, e^{i b}\right] \subset S^{1}$ with $0 \leq a<b \leq 2 \pi$, let $\sqrt{I}:=\left[e^{i a / 2}, e^{i b / 2}\right]$. The square root function induces an isomorphism $\mathcal{A}(I) \rightarrow \mathcal{A}(\sqrt{I})$. For $I \subset S^{1}, 1 \notin \stackrel{\mathrm{I}}{\text {, }}$ the action of $\mathcal{A}(I)$ on $H_{0}$ is the composite $\mathcal{A}(I) \rightarrow \mathcal{A}(\sqrt{I}) \rightarrow B\left(H_{0}\right)$ of the above isomorphism with the standard action of $\mathcal{A}(\sqrt{I})$ on $H_{0}$.

The equivalence between the above two descriptions of $\Omega_{\mathcal{A}}$ is provided by the linear map $L^{2}(\mathcal{A}(\sqrt{\cdot})): L^{2}\left(\mathcal{A}\left(S_{\text {cut }}^{1}\right)\right) \rightarrow L^{2}\left(\mathcal{A}\left(\left[e^{i 0}, e^{i \pi}\right]\right)\right)=H_{0}$.

Theorem 9. Let $\mathcal{A}$ be a non-trivial conformal net. Then $\Omega_{\mathcal{A}} \in \mathcal{T}_{\mathcal{A}}$ is characterized up to isomorphism by the following three properties:

(a) it is non-zero,

(b) $\Omega_{\mathcal{A}} \oplus \Omega_{\mathcal{A}} \cong \Omega_{\mathcal{A}}$, and

(c) the actions of $\mathcal{A}(I)$ for $I \subset S^{1}, 1 \notin \stackrel{\circ}{I}$, factor through an action of $\mathcal{A}\left(S_{\text {cut }}^{1}\right)$. Moreover, if $\mathcal{A}$ has finite index, then $\Omega_{\mathcal{A}}$ is an absorbing object.

Proof. We first check that $\Omega_{\mathcal{A}}$ satisfies the above three properties. The first one is obvious. The third one holds by construction. The second property is a consequence of Lemma 10 below: since $\mathcal{A}\left(S_{\text {cut }}^{1}\right)$ is an infinite factor, the Hilbert spaces $L^{2}\left(\mathcal{A}\left(S_{\text {cut }}^{1}\right)\right) \oplus L^{2}\left(\mathcal{A}\left(S_{\text {cut }}^{1}\right)\right)$ and $L^{2}\left(\mathcal{A}\left(S_{\text {cut }}^{1}\right)\right)$ are isomorphic as left $\mathcal{A}\left(S_{\text {cut }}^{1}\right)$-modules. It follows that $\Omega_{\mathcal{A}} \oplus \Omega_{\mathcal{A}} \cong \Omega_{\mathcal{A}}$.

Let $\Omega^{\prime}$ be another soliton that satisfies the same three properties. Then $\Omega^{\prime}$ is naturally an $\mathcal{A}\left(S_{\text {cut }}^{1}\right)$-module. By the classification of modules over factors, there is a unique non-zero $\mathcal{A}\left(S_{\text {cut }}^{1}\right)$-module (up to isomorphism) that satisfies $\Omega^{\prime} \oplus \Omega^{\prime} \cong \Omega^{\prime}$. It follows that $\Omega^{\prime} \cong \Omega_{\mathcal{A}}$.

Let us now assume that $\mathcal{A}$ has finite index. Given a non-zero soliton $X$, we need to show that $X \otimes \Omega_{\mathcal{A}} \cong \Omega_{\mathcal{A}}$. Equivalently, we need to show that $X \otimes \Omega_{\mathcal{A}}$ satisfies the three properties listed above. The first property, $X \otimes \Omega_{\mathcal{A}} \neq 0$, holds because fusing over a factor sends non-zero Hilbert spaces to non-zero Hilbert spaces (see, e.g., [1, Prop. 5.2]). The second property, $X \otimes \Omega_{\mathcal{A}} \oplus X \otimes \Omega_{\mathcal{A}} \cong X \otimes \Omega_{\mathcal{A}}$, is an immediate consequence of the corresponding property of $\Omega_{\mathcal{A}}$. The third property is more tricky and its verification will occupy the rest of this proof.

Let $\mathcal{I}_{0}$ be the collection of all subintervals of $S^{1}$ whose interior does not contain the base point $1 \in S^{1}$. Equivalently, $\mathcal{I}_{0}$ is the collection of all subintervals $I \subsetneq S_{\text {cut }}^{1}$. By definition, a soliton is a Hilbert space equipped with compatible actions of all 
the algebras $\mathcal{A}(I)$ for $I \in \mathcal{I}_{0}$. Let $I_{1}:=\left[e^{i 0}, e^{i \pi / 2}\right]$ and $I_{4}:=\left[e^{i 3 \pi / 2}, e^{i 2 \pi}\right]$ be the first and fourth quadrants of the standard circle, and let $I_{14}:=I_{1} \sqcup I_{4} \subset S_{\text {cut }}^{1}$ be their disjoint union (whereas $I_{1}$ and $I_{4}$ are not disjoint in $S^{1}$, these intervals are disjoint when viewed as subsets of $S_{\text {cut }}^{1}$ ). The collection $\mathcal{I}_{0}$ is not a 2 -cover of $S_{\text {cut }}^{1}$, because no element of $\mathcal{I}_{0}$ contains $\partial S_{\text {cut }}^{1}$. But

$$
\mathcal{I}:=\mathcal{I}_{0} \cup\left\{I_{14}\right\}
$$

is a 2 -cover.

Recall that, by definition, $X \otimes \Omega_{\mathcal{A}}=X \otimes_{\mathcal{A}\left(I_{+}\right)} \Omega_{\mathcal{A}}$. By the split property, the actions of $\mathcal{A}\left(I_{+}\right)$and of $\mathcal{A}\left(I_{4}\right)$ on $\Omega_{\mathcal{A}}$ extend to an action of their spatial tensor product. Equivalently, there exists an intermediate type $I$ factor:

$$
\left.\mathcal{A}\left(I_{4}\right) \subset N \subset \mathcal{A}\left(I_{+}\right)^{\prime} \quad \text { (commutant inside } B\left(\Omega_{\mathcal{A}}\right)\right) .
$$

The action of $N$ on $\Omega_{\mathcal{A}}$ commutes with that of $\mathcal{A}\left(I_{+}\right)$and thus induces an action on $X \otimes_{\mathcal{A}\left(I_{+}\right)} \Omega_{\mathcal{A}}$. The latter commutes with the action of $\mathcal{A}\left(I_{1}\right)$ coming from $X$, so we get an intermediate type $I$ factor:

$$
\left.\mathcal{A}\left(I_{4}\right) \subset N \subset \mathcal{A}\left(I_{1}\right)^{\prime} \quad \text { (commutant inside } B\left(X \otimes_{\mathcal{A}\left(I_{+}\right)} \Omega_{\mathcal{A}}\right)\right) .
$$

Equivalently, the actions of $\mathcal{A}\left(I_{1}\right)$ and $\mathcal{A}\left(I_{4}\right)$ on $X \otimes_{\mathcal{A}\left(I_{+}\right)} \Omega_{\mathcal{A}}$ extend to their spatial tensor product $\mathcal{A}\left(I_{14}\right)$.

The above action of $\mathcal{A}\left(I_{14}\right)$ on $X \otimes \Omega_{\mathcal{A}}$, together with the actions of $\mathcal{A}(I)$ for $I \in \mathcal{I}_{0}$ coming from the fact that $X \otimes \Omega_{\mathcal{A}}$ is a soliton, assemble to a compatible family of actions

$$
\rho_{I}: \mathcal{A}(I) \rightarrow B\left(X \otimes \Omega_{\mathcal{A}}\right) \quad \forall I \in \mathcal{I} .
$$

Finally, by Lemma [6 since $\mathcal{I}$ is a 2-cover, these extend to an action of $\mathcal{A}\left(S_{\text {cut }}^{1}\right)$. This finishes the proof of condition $(c)$.

\section{ApPendix}

For classical conformal nets, it is well known that, unless $\mathcal{A}(I)=\mathbb{C}$ for all $I$, the algebras $\mathcal{A}(I)$ are hyperfinite $I I I_{1}$ factors [6, Thm. 2.13]. Hyperfiniteness is a formal consequence of the split property, and holds equally well in the coordinate free setup (i.e., for conformal net as in [2, Def. 1.1]). Indeed, given an interval $I$, write $\stackrel{\circ}{I}=\bigcup I_{n}$ with $I_{n} \subset \stackrel{\circ}{I}_{n+1}$. By the split property, there exist intermediate type $I$ subfactors $\mathcal{A}\left(I_{n}\right) \subset N_{n} \subset \mathcal{A}\left(I_{n+1}\right)$, and so $\mathcal{A}(I)=\bigvee N_{n}$ is hyperfinite.

We do not know how to prove the type $I I I_{1}$ property in the coordinate free setup. The following lemma is the best we can offer:

Lemma 10. Let $\mathcal{A}$ be a non-trivial conformal net. Then the algebras $\mathcal{A}(I)$ are infinite factors (they are infinite dimensional, and they are not of type $I I_{1}$ ).

Proof. The algebra $\mathcal{A}(I)$ is infinite dimensional as it contains infinitely many nontrivial commuting subalgebras.

Let $I_{0}$ be the upper half of the standard circle, so that the vacuum sector $H_{0}$ is $L^{2}\left(\mathcal{A}\left(I_{0}\right)\right)$. Assume by contradiction that the algebra $\mathcal{A}\left(I_{0}\right)$ is of type $I I_{1}$. Then the von Neumann dimension of $H_{0}$ as an $\mathcal{A}\left(I_{0}\right)$-module is equal to 1 . By diffeomorphism covariance, for every interval $I \subset S^{1}$, the dimension of $H_{0}$ as an $\mathcal{A}(I)$-module is also 1. Given two intervals $I \subsetneq J \subset S^{1}$, we have

$$
\operatorname{dim}_{\mathcal{A}(I)}\left(H_{0}\right)=[\mathcal{A}(J): \mathcal{A}(I)] \cdot \operatorname{dim}_{\mathcal{A}(J)}\left(H_{0}\right) .
$$


It follows that $[\mathcal{A}(J): \mathcal{A}(I)]=1$. The inclusion $\mathcal{A}(I) \rightarrow \mathcal{A}(J)$ is therefore an isomorphism, a contradiction.

\section{Acknowledgements}

This project has received funding from the European Research Council (ERC) under the European Union's Horizon 2020 research and innovation programme (grant agreement No 674978).

\section{REFERENCES}

[1] A. Bartels, C. Douglas and A. Henriques. Dualizability and index of subfactors. Quantum Topology, 5 (2014), no 3, 289-345.

[2] A. Bartels, C. Douglas and A. Henriques. Conformal nets I: Coordinate-free nets. Int. Math. Res. Not. IMRN no. 13 (2015), 4975-5052.

[3] A. Bartels, C. Douglas and A. Henriques. Conformal nets II: Conformal blocks. arXiv:1409.8672 (2014)

[4] K. Costello and O. Gwilliam. Factorization algebras in quantum field theory. to be published by Cambridge University Press.

[5] K. Fredenhagen, M. Jörß. Conformal Haag-Kastler nets, pointlike localized fields and the existence of operator product expansions. Comm. Math. Phys. 176 (1996), no. 3, 541-554.

[6] F. Gabbiani and J. Fröhlich. Operator algebras and conformal field theory. Comm. Math. Phys., 155(3):569-640, 1993.

[7] A. Guichardet. Sur la catégorie des algèbres de von Neumann. Bull. Sci. Math. 90 (1966), no. $2,41-64$.

[8] A. Henriques. What Chern-Simons theory assigns to a point. arXiv:1503.06254 (2015).

[9] A. Henriques and D. Penneys. Bicommutant categories from fusion categories. accepted in Selecta Mathematica (2016).

[10] A. Henriques. The classification of chiral WZW models by $H_{+}^{4}(B G, \mathbb{Z})$. accepted in Contemporary Mathematics (2016).

[11] A. Kornell. Quantum collections. arXiv:1202.2994 (2012).

[12] R. Longo and F. Xu. Topological sectors and a dichotomy in conformal field theory. Comm. Math. Phys., 251 (2004), no. 2, 321-364.

[13] M. Takesaki. Theory of operator algebras I. Encyclopaedia of mathematical sciences, 124, Operator algebras and non-commutative geometry V, Springer-Verlag (2002).

[14] V. Toledano Laredo. Fusion of positive energy representations of $\operatorname{LSpin}_{2 n}$. Ph.D. thesis, St. John's College, Cambridge, 1997.

[15] A. Wassermann. Operator algebras and conformal field theory. III. Fusion of positive energy representations of $L S U(N)$ using bounded operators. Invent. Math., 133 (1998), no. 3, 467538.

[16] F. Xu. Jones-Wassermann subfactors for disconnected intervals. Commun. Contemp. Math., 2 (2000), no. 3, 307-347. 